\title{
Hepatitis B Virus (HBV) and Hepatitis C Virus (HCV) Infections among Hemodialysis Patients
}

\section{Abdulrahman A. Alkhan*}

Specialist Epidemiologist and Infection Control Director, Dhahran General Hospital, Saudi Arabia

"Corresponding author: Dr. Abdulrahman A. Alkhan, Specialist Epidemiologist and Infection Control Director, Dhahran General Hospital, first street 2 b, Dhahran, Eastern Province 31452, Saudi Arabia, Tel: 00966138092090; Fax: 00966138915619; E-mail: alkhanabdulrahman@yahoo.com

Rec date: Dec 14, 2014 Acc date: Jan 25, 2015 Pub date: Jan 29, 2015

Copyright: ( 2015 Alkhan AA. This is an open-access article distributed under the terms of the Creative Commons Attribution License, which permits unrestricted use, distribution, and reproduction in any medium, provided the original author and source are credited.

\begin{abstract}
Back ground: HBV is a global health problem which affects about 2 billion people worldwide; it is a chronic disease in $15 \%$ of adult population. HCV is also a global health problem which effects about 200 million people worldwide, $3 \%$ of world population are living with chronic hepatitis $C$ and about $3-4$ million people are infected each year, and about 350,000 people die every year due to HCV.
\end{abstract}

Hemodialysis is an artificial way of maintaining hemostasis in the body in the patients with severe renal impairment; it is indicated for the patient with acute renal failure and stage IV chronic renal failure, most of the patients who are undergoing dialysis do that for long period of times and are exposed to the numerous side effects.

One of these side effects is infection with hepatitis B and C It was recognized since 1977 the hazards of HBV infection to the hemodialysis patients and since 1990 for HCV. Several studies are done worldwide regarding the incidence, prevalence and risk factors of HBV and HCV infections to the hemodialysis patient.

The prevalence of HBV and HCV infections varies greatly from country to country, with $14 \%$ of hemodialysis patients in Saudi Arabia being hepatitis B positive and 7\% of them being hepatitis C positive. In other Arab countries $23.7 \%$ in Sudan, $71 \%$ in Kuwait and $41 \%$ in Tunisia, Egypt the prevalence of hepatitis $C$ in hemodialysis patient ranges from 52.3 to $82 \%$.

Objectives: To estimate the prevalence of HBV and HCV infections among hemodialysis patients. To determine the risk factors of hepatitis $\mathrm{B}$ and $\mathrm{C}$ infection in hemodialysis patients. To assess Level of awareness of the patients and health care workers about the HBV and HCV infections.

Conclusion: Among all risk factors were studied, only the long duration of hemodialysis was significantly associated with HBV and HCV positivity, suggesting that HBV and HCV were nosocomial transmission and the nonadherence to the known universal infection control precautions could be contributing to the high prevalence. Education of staff members and adhere strictly to universal infection control precautions remains a cornerstone for prevention of hepatitis transmission among patients undergoing maintenance hemodialysis.

Recommendations: Preventing transmission of HBV and HCV and other blood borne viruses among hemodialysis patients from both recognized and unrecognized sources of infection requires implementation of a comprehensive infection control program. Introduce training courses in infection control for all health care workers at being of work and annually. Vaccinate all patients and staff against HBV.

Keywords: HBV and HCV; Infections; Hemodialysis; Risk factors; Prevalence; Prevention

\section{Characteristic of $\mathrm{HBV}$ and $\mathrm{HCV}$ and their Infection [1-4]}

\section{Virology}

HBV is divided into eight genotypes (A-H) also four major serotypes (adr, adw, ayr, ayw). HCVin comparison, there are seven major genotypes of $\mathrm{HCV}$, which are indicated numerically from one to seven $[7,8]$.

\section{Mode of Transmission}

The infection can be transmitted through different routes. HBV, $\mathrm{HCV}$ are readily detected in serum. It is seen at very low levels in semen, vaginal mucus, saliva, and tears (Figure 1).

The virus is not detected in urine, stool, or sweat [9]. It is well documented that hemodialysis patients Have higher rates of HBV, HCV infection, which proved by many studies done worldwide (Table $1)$. 
Citation: Alkhan AA (2015) Hepatitis B Virus (HBV) and Hepatitis C Virus (HCV) Infections among Hemodialysis Patients. General Med 3:

Page 2 of 5

\begin{tabular}{|l|l|l|l|l|l|l|}
\hline Type & Transmission & Classification & Genome & Antigens & Incubation period & Chronicity \\
\hline HBV & Parenteral & Hepadnavirus & +dsDNA & HBsAg,HBeAg & $45-160$ days & Yes (uncommon) \\
\hline HCV & Parenteral & Hepacivirus & +ssRNA & Core antigen & $15-150$ days & Yes (common) \\
\hline
\end{tabular}

Table 1: Characteristic of HBV and HCV and their infection.

Studies conclude that the transmission of is generally nosocomial with possible risk factors such as in proper disinfecting of devices between patients, sharing of single- use vials for infusion, poor sterile technique, poor cleaning of dialysis machines, and poor distance between chairs [9].

\section{Epidemiology}

Intravenous drug use: is the main method of transmission of $\mathrm{HCV}$ in developed countries $[9,10]$.

Body piercings and tattooing: is associated with two to three fold increased risk of hepatitis C. This can be due to either improperly sterilized equipment or contamination of the dyes used [10] (Figure 1-4).

Shared personal care items such as razors, toothbrushes, and manicuring or pedicuring equipment can be contaminated with blood. Sharing such items can potentially lead to exposure to HCV [10].

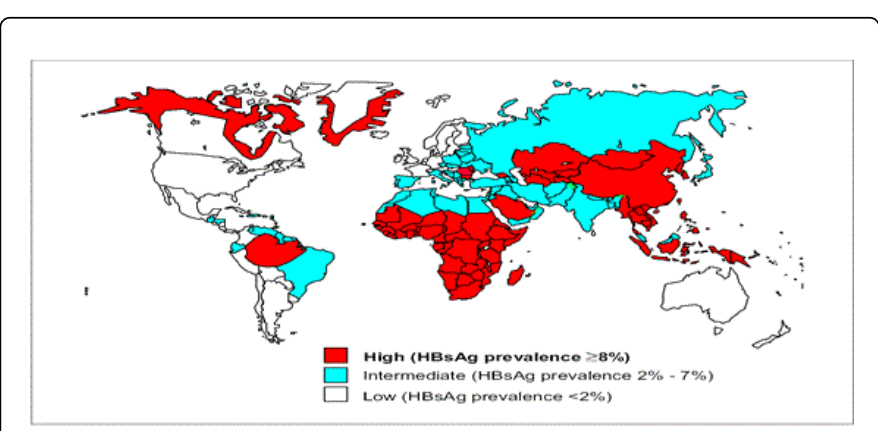

Figure 1: The prevalence of HBV worldwide [5]

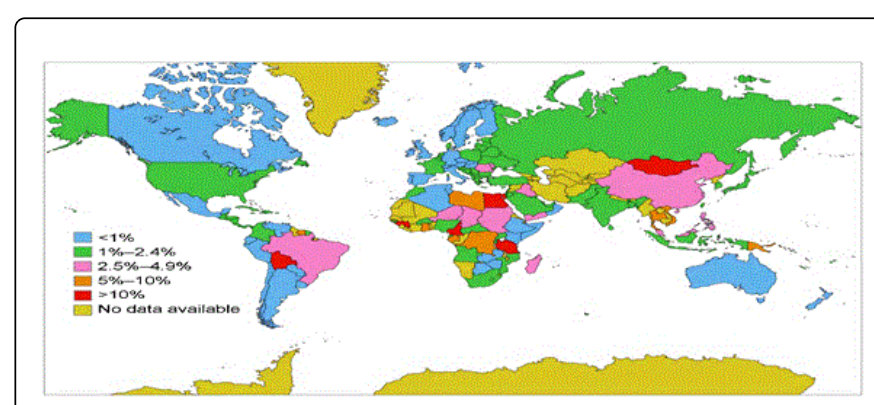

Figure 2: The prevalence of HCV world wide [6]
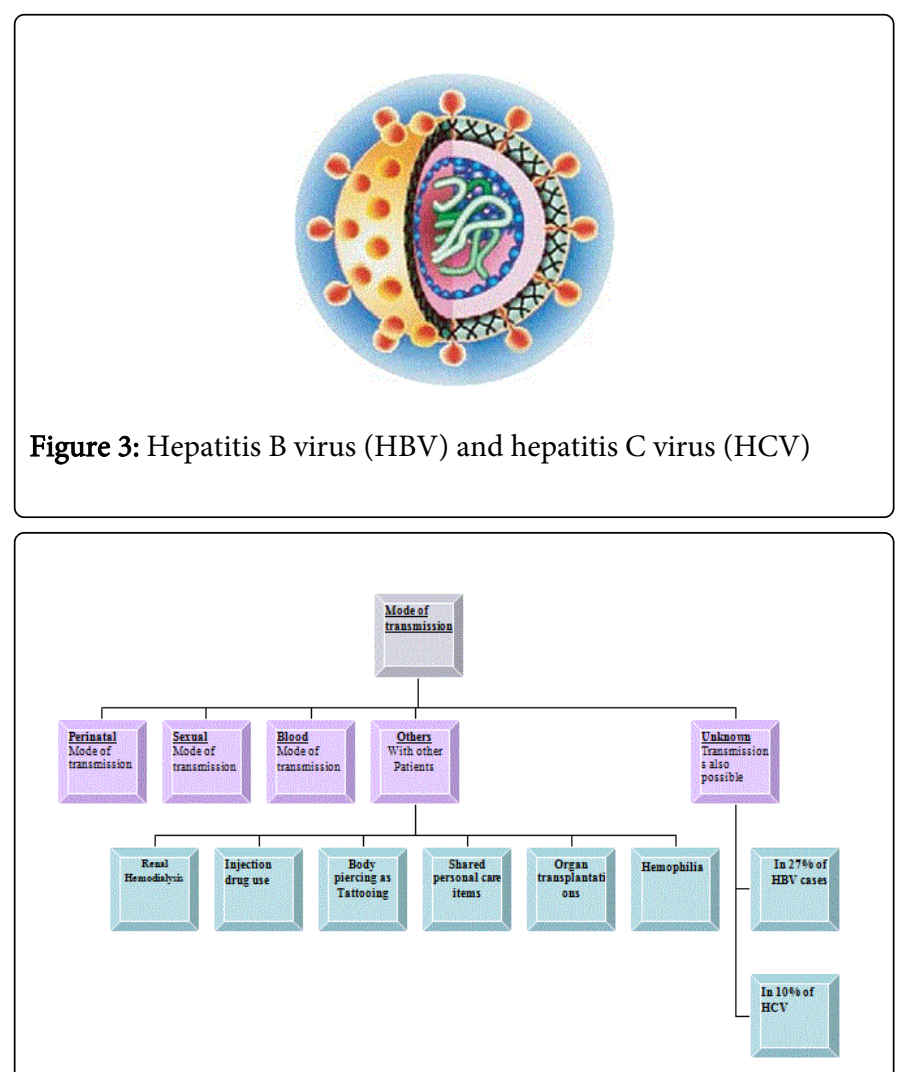

Figure 4: Mode of transmission

\section{Prevention}

Immunization: against HBV by Recombinant vaccines became available in the mid-1980s. However; there is no vaccine yet for HCV [11].

For risk groups such as hemodialysis patients, immunization is the most effective and cost-saving means of prevention [11].

- The vaccine is administered in two, three or four dose schedules into infants and adults, which provides protection for $85-90 \%$ of individuals.

- Education of high risk groups and health care personnel to reduce the risk of transmission to others.

- Screening of blood and blood products.

- Avoid sharing drug needles and unsanitary piercing methods. 


\section{Treatment}

HBV can be treated by antiviral drugs such as lamivudine, adefovir and the immune system modulators interferon alpha-2a and interferon- $a-2 b$ and pegylated interferon alpha- $2 a$ and $2 b$.

HCV the Current treatment is a combination pegylated interferon alpha and the antiviral drug ribavirin for a period of 24 or 48 weeks [11].

\section{Dialysis}

A process of removing waste and excess water from the blood, and is used primarily to provide an artificial replacement for lost kidney function. Dialysis works on the principles of the diffusion of solutes and ultra-filtration of fluid across a semi-permeable membrane.

\section{Prescription}

A prescription for dialysis by nephrologists will specify various parameters for a dialysis treatment. These include frequency (how many treatments per week), length of each treatment, and the blood and dialysis solution flow rates, as well as the size of the dialyzer. The composition of the dialysis solution is also sometimes adjusted in terms of its sodium and potassium and bicarbonate levels. In general, the larger the body size of an individual, the more dialysis he/she will need. 3-4 hour treatments (sometimes up to 5 hours for larger patients) given 3 times a week are typical. Twice-a-week sessions are limited to patients who have a substantial residual kidney function Four sessions per week are often prescribed for larger patients, as well as patients who have trouble with fluid overload [12].

\section{Indications of Dialysis}

These can be divided into acute or chronic indications. [13]:

Indications for dialysis in the patient with acute kidney injury are

- Acidemia from metabolic acidosis in situations in which correction with sodium bicarbonate is impractical or may result in fluid overload.

- Electrolyte abnormality, such as severe hyperkalemia.

- Overload of fluid not expected to respond to treatment with diuretics.

- Uremia complications, such as pericarditis, encephalopathy, or gastrointestinal bleeding.

\section{Chronic indications for dialysis:}

- Symptomatic renal failure.

- Low glomerular filtration rate (GFR) (renal replacement therapy often recommended to commence at a GFR of less than 10-15 $\mathrm{ml} / \mathrm{min} / 1.73 \mathrm{~m}^{2}$ ).

- Difficulty in medically controlling fluid overload, serum potassium, and/or serum phosphorus when the GFR is very low.

\section{Complication of Dialysis}

Complications that occur during a hemodialysis sessions are Hypotension (20-30\% of dialysis), Muscle cramps (5-20\%), Nausea and vomiting (5-15\%), Headache (5\%), Chest and back pain (2-5\%), Itching (5\%), Fever and chills $(<1 \%)$, Cardiopulmonary arrest, transmission of infections, like hepatitis and HIV. First Use Syndrome is a rare but severe anaphylactic reaction to the new dialyzer. Its symptoms include sneezing, wheezing, shortness of breath, back pain, chest pain, or sudden death. It can be caused by residual sterilant in the artificial kidney or the material of the membrane itself. In recent years, the incidence of First Use Syndrome has decreased, due to an increased use of gamma irradiation, steam sterilization, or electronbeam radiation instead of chemical sterilants, and the development of new semi permeable membranes of higher biocompatibility.

\section{Dialysis Disequilibrium Syndrome:}

- It is a common neurologic complication seen in dialysis patients that is characterized by weakness, dizziness, headache, and in severe cases, mental status changes. It is due to rapid flux in osmolality with hemodialysis.

- Mechanical complications which include are air embolism, hemolysis, blood leaks, and contaminated dialysate.

- Complications due to vascular access which include infection at the site of AV fistula, stenosis of fistula, hemorrhage, thrombosis, inflammatory complications, aneurysms or pseudoaneurysms.

\section{Prevalence of HBV and HCV Infections among Hemodialysis Patients}

Infection with hepatitis among hemodialysis patients was an issue for years. The first published recommendations for prevention of HBV infection among hemodialysis patients was published in United States in 1977. By 1980, the concepts of hand hygiene, education of health care providers and catheter care were widely emphasized and this led to sharp reduction in the incidence of hepatitis B among hemodialysis patients. As for hepatitis C, it was discovered in 1988 and it is prevention measures were implemented in 1990. The prevalence of hepatitis attributed to dialysis was declining in the past decade, but it varies among countries and among regions. The prevalence is from the low $1.9 \%$ in Slovenia to high $80 \%$ in Senegal.

In developing countries the prevalence is high as showed by studies done in Taiwan, the prevalence was $16.1 \%$. In Pakistan prospective cross-sectional study at the dialysis unit of Sindh Institute of Urology in 2010 showed that among 1220 patients going through hemodialysis. 124 were hepatitis positive (prevalence at $10.2 \%$ ). In Kenya a Crosssectional descriptive study was conducted in 2003 at Kenyatta national hospital, among 100 patients undergoing dialysis, hepatitis B was found in 8 patients (8\%) and hepatitis C in 5 patients (5\%). In India study by Christian medical college in 2001 from the all patients who undergoing dialysis at the time of the study $29 \%$ of them were hepatitis B positive and about $20 \%$ were hepatitis $C$ positive. In Arab countries $23.7 \%$ in Sudan, $71 \%$ in Kuwait and $41 \%$ in Tunisia, Egypt the prevalence of $\mathrm{HCV}$ in hemadalysis patient ranges from 52.3 to $82 \%$.

In a joint study by Saudi Arabia and Bahrain in 2004 showed 7.4\% of hemodialysis patient were HCV positive compared to $14.7 \%$ how $\mathrm{HBV}$ were positive. In developed countries in USA study showed only $0.3 \%$ of the patient were HCV positive. In Canada 1998 study done in Alberta and showed that hepatitis $\mathrm{C}$ infection in hemodialysis patients were $6.5 \%$ and $\mathrm{HBV}$ were only $1.2 \%$ [13].

\section{Risk Factors of HBV and HCV Infections in Hemodialysis Patients}

The understanding of the risk of transmission of hepatitis B and C among haemadialysis patients is essential to undertake the appropriate 
Page 4 of 5

measures to prevent its transmission. A number of risk factors are implicated including blood transfusion, duration of dialysis, dialysis machine sterilization and preparation and the use of common medication carts. Studies conclude that the transmission of Virus to haemodialysis patients is generally nosocomial with possible risk factors being failure to disinfect devices between patients, sharing of single- use vials for infusion, poor sterile technique, poor cleaning of dialysis machines, and poor distance between chairs [14].

\section{General Hygiene Violation}

The number one attributed risk factor for reduction of incidence is general hygienic measures. In April 1994 an outbreak of HBV occurred in 5 hemadalysis centers in California, Nebraska and Texas, US (CDC el 1996). The cause of the outbreak was thought to be due to failure of identification and isolation of a patient with $\mathrm{HBV}$.

\section{Blood Transfusion}

It is the most important risk factors. a retrospective study in Sweden with 236 patients in dialysis center and 23 patients who became sero positive $\mathrm{HCV}$ in the period of study, $80 \%$ of them had blood transfusion. In other study in Gaza they found strong relationship between the number of transfused blood units and HBV and HCV infections, results showed that the more units of blood the patients received, the higher the incidence of hepatitis infection [15].

\section{Duration of Dialysis}

One of the most challenging things is to separate that if the long duration of the dialysis is a risk factor by it is own or because the longer the period the more blood transfusion the patient will have. It was found in a study done by CDC (center for disease control) that patient who had dialysis less than 5 years $12 \%$ of them has hepatitis $\mathrm{C}$ and $37 \%$ of the patient who had the dialysis more than 5 years.

\section{Prevention of Hepatitis Transmission}

One of the most important strategies of prevention is management of anemia in hemodialysis patient. Anemia is common in patients with chronic kidney disease. The landmark study by Obrador et al., showed that among predialysis patients, $68 \%$ of those with advanced chronic kidney disease who required renal replacement therapy had a hematocrit less than $30 \mathrm{mg} / \mathrm{dL}$; of these, $51 \%$ of patients had a hematocrit less than $28 \mathrm{mg} / \mathrm{dL}$. Furthermore, although anemia is not as common in earlier stages of chronic kidney disease, patients with stage III disease have a prevalence of concurrent anemia of $5.2 \%$, whereas those with stage IV disease have a prevalence of concurrent anemia of $44.1 \%$ [15].

The patient with chronic renal disease and end stage renal disease has anemia due to Erythropoietin deficiency. Early anemia identification and treatment will substantially reduce the need for urgent blood transfusion, also iron supplementation is recommended. Another aspect of infection control is practicing safety guidelines of general hygiene. There are guidelines published by CDC (center for disease control) in 1988, also there is standardized measures that the dialysis machine should washed by $2.5 \mathrm{~L}$ of normal saline and then it will be disinfected by chloroform and acetic acid or citric acid solution.

Immunization against HBV by Recombinant vaccines became available in the mid-1980s. However, there is no vaccine yet for HCV. For risk groups such as haemodialysis is most effective and cost-saving means of prevention. The vaccine is administered in two, three or four dose schedules into infants and adults, which provides protection for $85-90 \%$ of individuals [16].

\section{Recommendations}

Preventing transmission of HBV and HCV and other blood borne viruses among hemodialysis patients from both recognized and unrecognized sources of infection requires implementation of a comprehensive infection control program. The components of such a program include infection control practices specifically designed for the hemodialysis setting (Table 2). Introduce training courses in infection control for all health care workers at being at work and annually, or at least before they being working in unit

\footnotetext{
Infection Control Precautions for haemodialysis units [17,18].

*Wear disposable gloves when caring for the patient or touching the patient's equipment at the dialysis station; remove gloves and wash hands between each patient or station.

*Items taken into the dialysis station should be disposed of, dedicated for use only on a single patient, or cleaned and disinfected before taken to a common clean area or used on another patient.

"Non disposable items that cannot be cleaned and disinfected (e.g., adhesive tape, cloth-covered blood pressure cuffs) should be dedicated for use only on a single patient.

*Unused medications (including multiple dose vials containing diluents) or supplies (syringes, alcohol swabs, etc.) taken to the patient's station should be used only for that patient and should not be returned to a common clean area or used on other patients.

"Do not use common medication carts to deliver medications to patients. Do not carry medication vials, syringes, alcohol swabs or supplies in pockets and should be brined prepared. If trays are used to deliver medications to individual patients, they must be cleaned between patients.

*Clean areas should be clearly designated for the preparation, handling and storage of medications and unused supplies and equipment. Clean areas should be clearly separated from contaminated areas where used supplies and equipment are handled. Do not handle and store medications or clean sup- plies in the same or an adjacent area to that were used equipment or blood samples are handled.

"Use external venous and arterial pressure transducer filters/protectors for each patient treatment to prevent blood contamination of the dialysis machines' pressure monitors. Change filters/protectors between each patient treatment, and do not reuse them. Internal transducer filters do not need to be changed routinely between patients.
} 
Citation: Alkhan AA (2015) Hepatitis B Virus (HBV) and Hepatitis C Virus (HCV) Infections among Hemodialysis Patients. General Med 3: 1000165. doi:10.4172/2327-5146.1000165

Page 5 of 5

\begin{tabular}{l} 
"Clean and disinfect the dialysis station (chairs, beds, tables, machines, etc.) between patients. \\
"Give special attention to cleaning control panels on the dialysis machines and other surfaces that are frequently touched and potentially contaminated with patients' \\
blood. \\
"Staff members caring for HBsAg-positive patients should not care for HBV susceptible patients at the same time (e.g., during the same shift or during patient change- \\
over). \\
\hline 'Discard all fluid and clean and disinfect all surfaces and containers associated with the prime waste (including buckets attached to the machines). \\
\hline
\end{tabular}

Table 2: Recommended infection control practices for hemodialysis units.

Training and education of patients (or family members for patients unable to be responsible for their own care) regarding infection control practices should be given on admission to dialysis and at least annually thereafter and should address the following topics: - Personal hygiene and hand washing technique and Patient responsibility for proper care of the access and recognition of signs of infection, which should be reviewed each time the patients has a change in access type. Vaccinate all patients and staff against HBV. Increase the number of the health care workers in haemodialysis units.

\section{References}

1. Maynard JE (1990) Hepatitis B: global importance and need for control. Vaccine 8 Suppl: S18-20.

2. el Guneid AM, Gunaid AA, O'Neill AM, Zureikat NI, Coleman JC, et al. (1993) Prevalence of hepatitis B, C, and D virus markers in Yemeni patients with chronic liver disease. J Med Virol 40: 330-333.

3. Margolis HS, Alter MJ, Hadler SC (1997) Viral hepatitis. In Evans AS, Kaslow RA (eds.) Viral infections of humans. Epidemiology and control (Fourth Edition). New York, Plenum Publishing Corporation 363-418

4. World Health Organization. Hepatitis B. WHO fact sheet204.2000.

5. Hou J, Liu Z, Gu F (2005) Epidemiology and Prevention of Hepatitis B Virus Infection. Int J Med Sci 2: 50-57.

6. Alter MJ, Hadler SC, Margolis HS, Alexander WJ, Hu PY, et al. (1990) The changing epidemiology of hepatitis B in the United States. Need for alternative vaccination strategies. JAMA 263: 1218-1222.

7. Toukan A (1997) Control of hepatitis B in the Middle East. In: Rizzetto M (ed.) Proceedings of IX Triennial International Symposium on Viral Hepatitis and Liver Disease. Turin, Edizioni Minerva Medica 678-679
8. Alexandria, World Health Organization Regional Office for the Eastern Mediterranean (1995) Intercountry workshop on the prevention and control of viral hepatitis.

9. al-Faleh FZ, Ayoola EA, Arif M, Ramia S, al-Rashed R, et al. (1992) Seroepidemiology of hepatitis B virus infection in Saudi Arabian children: a baseline survey for mass vaccination against hepatitis B. J Infect 24: 197-206.

10. Arya SC, Ashraf SJ, Parande CM, el-Sayed M, Sahay R, et al. (1985) Hepatitis B virus in Gizan, Saudi Arabia. J Med Virol 17: 267-274.

11. Awidi AS, Tarawneh MS, el-Khateeb M, Hijazi S, Shahrouri M (1984) Incidence of hepatitis B antigen among Jordanian volunteer blood donors. Public Health 98: 92-96.

12. el Goulli N, Coursaget P, Chiron JP, Kastally R, Ben Khaliffa H, et al. (1984) [Hepatitis B virus infection in Tunisia]. IARC Sci Publ : 199-211.

13. Elshafie SS (1992) The prevalence of hepatitis B surface antigen in the Gezira (Sudan). Afr J Med Med Sci 21: 61-63.

14. Parande CM, Arya SC, Ashraf SJ (1986) Hepatitis B virus among Saudi children in Gizan, Saudi Arabia. Infection 14: 223-225.

15. Ramia S, Hossain A, Bakir TM, Waller DK, Vivian PA (1986) Prevalence and subtype of hepatitis B surface antigen (HBsAg) in the Saudi population. Trop Geogr Med 38: 63-69.

16. Scott DA, Constantine NT, Callahan J, Burans JP, Olson JG, et al. (1992) The epidemiology of hepatitis $\mathrm{C}$ virus antibody in Yemen. Am J Trop Med Hyg 46: 63-68.

17. Soliman AT, Bassiouny MR, Elbanna NA (1995) Study of hepatic functions and prevalence of hepatitis-B surface antigenaemia in Omani children with sickle cell disease. J Trop Pediatr 41: 174-176.

18. Talukder MA, Gilmore R, Bacchus RA (1982) Prevalence of hepatitis B surface antigen among male Saudi Arabians. J Infect Dis 146: 446. 